The boundary conditions implied by conditions (i) and (ii) are:

For Eq. (4): (a) $u_{\theta}=0, u_{r}$ finite, when $\theta=0$.

(b) $u_{r}=u_{\theta}=0$, when $\theta=\alpha$.

For Eq. (5): (c) $u_{\theta}=0, u_{r}$ finite, $\partial u_{r} / \partial \theta$ finite, when $\theta=0 .^{*}$

These yield respectively the equations:

$$
\begin{gathered}
c_{1}+c_{2}+c_{3}=0, \\
c_{1} \cos ^{2} \alpha+c_{2} \cos \alpha+c_{3}=0, \\
2 c_{1}+c_{2}=0 .
\end{gathered}
$$

These have $c_{1}=c_{2}=c_{3}=0$ as their only solution.

As Squire [5] has shown, the general solution of Eq. (4) with $c_{1}=c_{2}=c_{3}=0$ is:

$$
f=\frac{2 \nu \sin ^{2} \theta}{a+1-\cos \theta}
$$

where $a$ is an arbitrary constant.

Referring again to the boundary condition $u_{r}(\alpha)=u_{\theta}(\alpha)=0$, we see that there is no finite value of $a(a=\infty$ yields a satisfactory but trivial solution) which satisfies this boundary condition, no matter what value of $\alpha$ is chosen.

Thus we have shown that there is no non-trivial solution of the form $\psi=r f(\theta)$ that is compatible with the Navier-Stokes equations and the boundary conditions (i) and (ii).

The author wishes to thank Prof. Garrett Birkhoff for suggesting the problem and for his helpful advice.

\title{
REFERENCES
}

[1] S. Goldstein (ed.), Modern developments in fluid dynamics, vol. 1, Oxford, 1938, p. 147. Also H. Schlichting, Grenzschichtstheorie, Karlsruhe

[2] H. B. Squire, Phil. Mag. 43, 942-5 (1952)

[3] V. L. Yatseev, Zh. eksp. teor. fiz. 20, 1031-34 (1950)

[4] S. Goldstein (ed.), Modern developments in fluid dynamics, vol. 1, Oxford, 1938, pp. 104 and 115

[5] H. B. Squire, Quart. J. Mech. Appl. Math. 4, 321-29 (1950)

*It is assumed that when these boundary conditions are substituted in Eqs. (4) and (5) that the limit $\theta \rightarrow 0$ is taken, since $\theta=0$ is a singular point in the spherical polar coordinate system.

\section{HEAT CONDUCTION IN SEMI-INFINITE SOLID IN CONTACT WITH LINEARLY INCREASING MASS OF FLUID*}

\author{
By C. C. CHAO AND J. H. WEINER (Columbia University)
}

Introduction. Problems of transient heat conduction in which the surface of a solid is in contact with a well-stirred fluid have been the subject of numerous investigations. ${ }^{1}$ In all previous cases studied, the mass of the fluid has been considered constant. However, it is sometimes of interest to know the temperature in the solid and fluid

*Received August 9, 1955.

${ }^{1} \mathrm{~A}$ review of previous work is found in [-1], pp. 16-17. 
while the latter is being poured onto the solid. This is so, for example, in the analysis of the filling stage of a casting process.

In this paper, the problem of a semi-infinite solid in perfect contact with a wellstirred fluid whose mass is initially zero and increases linearly with time is considered. The principal boundary condition involved has variable coefficients and the solution of the problem by the Laplace transform technique requires the solution of a differential equation with the Laplace transform parameter as independent variable. Numerical results are presented.

Problem. The problem described above is formulated mathematically as follows. (Property values are taken independent of temperature; the initial temperature of the solid is taken as zero.)

$$
\begin{gathered}
\kappa \frac{\partial^{2} u}{\partial x^{2}}=\frac{\partial u}{\partial t} ; \quad x>0, \quad t>0, \\
\lim _{t \rightarrow 0} u(x, t)=0 ; \quad x>0, \\
\lim _{x \rightarrow \infty} u(x, t)=0 ; \quad t \geq 0, \\
K \frac{\partial u}{\partial x}=m c\left[\frac{\partial}{\partial t}(t u)-V\right] ; \text { at } \quad x=0, \quad t>0,
\end{gathered}
$$

where $u(x, t)$ is the temperature in the solid at distance $x$ from the surface at time $t$ after the start of pour, $K$ is the thermal conductivity of the solid, $\kappa$ its thermal diffusivity, $V$ is the pouring temperature of the liquid, $c$ is the specific heat of the liquid, and $m$ is its (constant) mass rate of flow.

Equation (4) is based on the assumptions that the fluid is well-stirred and is in perfect contact with the solid, that is, that the entire mass of liquid is at the temperature of the solid surface. It is derived from a heat balance on the liquid.

Solution. The solution of the problem is obtained by use of the Laplace transform.

Let $U(x, p)=\int_{0}^{\infty} \epsilon^{-p t} u(x, t) d t$ denote the Laplace transform of $u(x, t)$. Then the Laplace transforms of Equations (1)-(4) are:

$$
\begin{gathered}
\frac{\partial^{2} U}{\partial x^{2}}=\frac{p U}{\kappa} ; \quad x>0, \\
\lim _{x \rightarrow \infty} U(x, p)=0, \\
K \frac{\partial U}{\partial x}=-m c\left[p \frac{\partial U}{\partial p}+\frac{V}{p}\right] ; \quad x=0 .
\end{gathered}
$$

The transform of Eq. (4), Eq. (7), is obtained by using the operational properties of the Laplace transform ([2], operations 3 and 8, p. 294).

The solution of Eq. (5) which satisfies Eq. (6) is:

$$
U=A(p) \exp \left[-(p / \kappa)^{1 / 2} x\right] .
$$


Substitution of Eq. (8) into Eq. (7) leads to the following differential equation for $A(p)$ :

$$
\frac{d A}{d p}-\frac{s A}{p^{1 / 2}}=\frac{V}{p^{2}},
$$

where $s=K / m c \kappa^{1 / 2}$.

The solution of Eq. (9) is

$$
A(p)=\frac{V}{p}-4 s^{2} V\left[\left(2 s p^{1 / 2}\right)^{-1}+\exp \left(2 s p^{1 / 2}\right) E i\left(-2 s p^{1 / 2}\right)\right]+C \exp \left(2 s p^{1 / 2}\right),
$$

where $E i(-x)=\int_{x}^{\infty} y^{-1} e^{-y} d y$ is the exponential integral. It is seen that the arbitrary constant, $C$, must be zero if $A(p)$ is to have a sectionally continuous inverse transform of exponential order.

Therefore, substitution of Eq. (10) with $C=0$ in Eq. (8) yields the transform of $u(x, t)$ as

$U(x, p)=V\left\{p^{-1}-4 s^{2}\left[\left(2 s p^{1 / 2}\right)^{-1}+\exp \left(2 s p^{1 / 2}\right) E i\left(-2 s p^{1 / 2}\right)\right]\right\} \exp \left[-(p / \kappa)^{1 / 2} x\right]$.

The following transform pair is known ([4], p. 268, pair 31):

$$
L^{-1}\left[(2 s)^{-1}+p_{。} \exp (2 s p) E i(-2 s p)\right]=(t+2 s)^{-2} .
$$

Therefore,

$L^{-1}\left\{\left[(2 s)^{-1}+p \exp (2 s p) E i(-2 s p)\right] \exp \left(-x p \kappa^{-1 / 2}\right)\right\}=\left(t-x \kappa^{-1 / 2}+2 s\right)^{-2}, t>x \kappa^{-1 / 2}$.

$$
=0, \quad t<x \kappa^{-1 / 2} \text {. }
$$

The following property of Laplace transforms is known ([1], p. 243): If

$$
L^{-1}[W(p)]=w(t),
$$

then

$$
L^{-1}\left[W\left(p^{1 / 2}\right) p^{-1 / 2}\right]=(\pi t)^{-1 / 2} \int_{0}^{\infty} w(y) \exp \left(-y^{2} / 4 t\right) d y
$$

therefore,

$$
\begin{aligned}
L^{-1}\left[\exp \left(2 s p^{1 / 2}\right) E i\left(-2 s p^{1 / 2}\right)+\left(2 s p^{1 / 2}\right)^{-1}\right] \exp \left[-(p / \kappa)^{1 / 2} x\right] \\
=(\pi t)^{-1 / 2} \int_{x / \kappa^{1 / 2}}^{\infty}\left(y-x \kappa^{-1 / 2}+2 s\right)^{-2} \exp \left(-y^{2} / 4 t\right) d y, \\
=\left(2 t \pi^{1 / 2}\right)^{-1} \int_{x / 2(\kappa t)^{1 / 2}}^{\infty}\left\{\xi-\left[x / 2(\kappa t)^{1 / 2}\right]+s t^{-1 / 2}\right\}^{-2} \exp \left(-\xi^{2}\right) d \xi .
\end{aligned}
$$

Use of the above transformation pair in Eq. (11) together with the known pair ([1], p. 381, pair 8)

$$
L^{-1}\left\{p^{-1} \exp \left[-(p / \kappa)^{1 / 2} x\right]\right\}=\operatorname{erfc}\left[x / 2(\kappa t)^{1 / 2}\right]
$$


in Eq. (11) yields

$$
\begin{aligned}
u(x, t)=V & {\left[\operatorname{erfc}\left[x^{\prime} 2(\kappa t)^{1 / 2}\right]\right.} \\
& \left.-2 s^{2} t^{-1} \pi^{-1 / 2} \int_{x / 2(\kappa t) \times / 2}^{\infty}\left\{\xi-\left[x / 2(\kappa t)^{1 / 2}\right]+s t^{-1 / 2}\right\}^{-2} \exp \left(-\xi^{2}\right) d \xi\right] .
\end{aligned}
$$

The above analysis has been purely formal. It may be verified, however, by direct substitution that the above function satisfies Eqs. (1)-(4) defining the boundary value problem. The interchange of differentiation and integration required in this verification may be readily justified.

Numerical results. The temperature of the liquid, which is equal to $u(0, t)$, is of particular interest. By use of integration by parts, it may be put in the following form:

$u(0, t)=V\left\{1-2 s(\pi t)^{-1 / 2}+2 s^{2} t^{-1}-4 s^{3}\left(\pi t^{3}\right)^{-1 / 2} \int_{0}^{\infty}\left(\xi+s t^{-1 / 2}\right)^{-1} \exp \left(-\xi^{2}\right) d \xi\right\}$.

Numerical values for the latter integral have been tabulated by Goodwin and Staton [3]. However, in the evaluation of Eq. (13), it was found necessary to carry their asymptotic expansion further in order to obtain sufficiently accurate results. A graph of $u(0, t) / V$ as function of $t / s^{2}$ is shown in Fig. 1 .

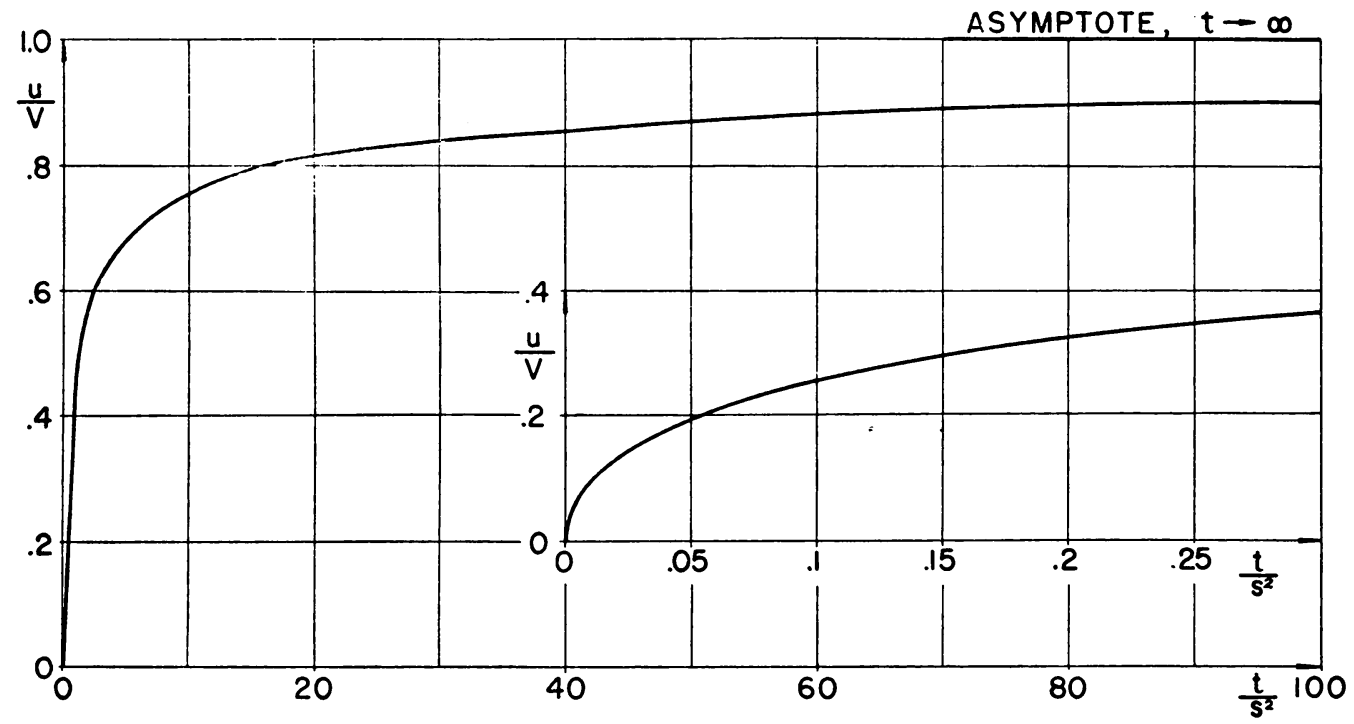

FIg. 1 Variation of fluid temperature with time.

\section{REFERENCES}

[1] H. S. Carslaw and J. C. Jaeger, Conduction of heat in solids, Oxford University Press, London, 1948

[2] R. V. Churchill, Modern operational mathematics in engineering, McGraw-Hill, New York, 1944

[3] E. T. Goodwin and J. Staton, Table of $\int_{0}^{\infty}(u+x)^{-1} \exp \left(-u^{2}\right) d u$, Quart. J. Mech. and Appl. Math., $1,220-224$ (1948)

[4] A. Erdelyi, Tables of integral transforms, vol. I, McGraw-Hill, New York, 1954 